\title{
The short GRB 070707 afterglow and its very faint host galaxy ${ }^{\star}$
}

\author{
S. Piranomonte ${ }^{1}$, P. D’Avanzo ${ }^{2}$, S. Covino ${ }^{2}$, L. A. Antonelli ${ }^{1}$, A. P. Beardmore ${ }^{3}$, S. Campana ${ }^{2}$, G. Chincarini ${ }^{2,4}$, \\ V. D'Elia ${ }^{1}$, M. Della Valle ${ }^{5,6,7}$, F. Fiore ${ }^{1}$, D. Fugazza ${ }^{2}$, D. Guetta ${ }^{1}$, C. Guidorzi ${ }^{2}$, G. L. Israel ${ }^{1}$, D. Lazzati ${ }^{8,9}$, \\ D. Malesani ${ }^{10}$, A. M. Parsons ${ }^{11}$, R. Perna ${ }^{8}$, L. Stella ${ }^{1}$, G. Tagliaferri ${ }^{2}$, and S. D. Vergani ${ }^{12,13}$
}

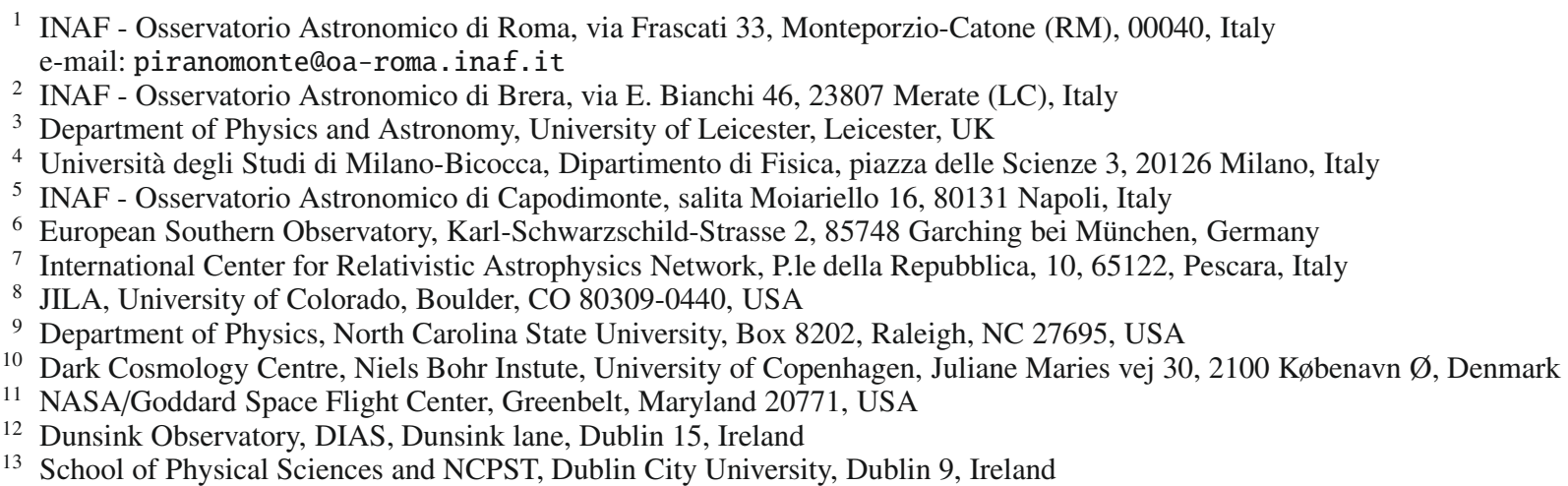

Received 8 July 2008 / Accepted 10 September 2008

\section{ABSTRACT}

\begin{abstract}
We present the results from an ESO/VLT campaign aimed at studying the afterglow properties of the short/hard gamma ray burst GRB 070707. Observations were carried out at ten different epochs from $\sim 0.5$ to $\sim 80$ days after the event. The optical flux decayed steeply with a power-law decay index greater than 3, later levelling off at $R \sim 27.3 \mathrm{mag}$; this is likely the emission level of the host galaxy, the faintest yet detected for a short GRB. Spectroscopic observations did not reveal any line features/edges that could unambiguously pinpoint the GRB redshift, but set a limit $z<3.6$. In the range of allowed redshifts, the host has a low luminosity, comparable to that of long-duration GRBs. The existence of such faint host galaxies suggests caution when associating short GRBs with bright, offset galaxies, where the true host might just be too dim for detection. The steepness of the decay of the optical afterglow of GRB 070707 challenges external shock models for the optical afterglow of short/hard GRBs. We argue that this behaviour might result from prolonged activity of the central engine or require alternative scenarios.
\end{abstract}

Key words. gamma rays: bursts

\section{Introduction}

Gamma-ray bursts (GRBs) are among the most powerful explosions in the universe. They are revealed in the hard X-ray/soft gamma-ray band and are followed in many cases by a fading afterglow observable from radio to X-ray wavelengths. GRBs are empirically classified in two groups (Mazets et al. 1981; Norris et al. 1984; Kouveliotou et al. 1993; Tavani 1996): short GRBs last less than $2 \mathrm{~s}$ and have a hard spectrum; long GRBs have longer durations (typically tens to hundreds seconds) and somewhat softer spectra. The emergence of a typical supernova ( $\mathrm{SN})$ spectrum superposed on the rapidly decaying non-thermal afterglow weeks after the events and the association with blue, highly star-forming galaxies provided strong evidence that a significant fraction of long GRBs originates in the gravitational collapse of massive stars (but see Della Valle et al. 2006; Fynbo et al. 2006; Gal-Yam et al. 2006a).

Short GRBs are revealed less frequently than long GRBs (they comprise about $1 / 4$ and $1 / 10$ of the BATSE and Swift samples, respectively; Kouveliotou et al. 1993; Berger 2007);

^ Based on observations made with ESO Telescopes at the La Silla Paranal Observatory under program ID 079.D-0909. moreover their afterglows are weaker and, thus, more difficult to detect and follow up. These are among the reasons why the origin of short GRBs is still under debate, despite the important progresses made in the Swift era. The tight upper limits on any associated SN (Hjorth et al. 2005; Covino et al. 2006; Kann et al. 2008) as well as the association with a broad variety of Hubble types hosts, from elliptical (Berger et al. 2005) to moderate star forming galaxies (e.g. Covino et al. 2006; Fox \& Mészáros 2006; Berger 2008), rule out the core-collapse mechanism as the main channel for short-GRBs production and strongly suggest that the explosion mechanism and/or progenitors of short GRBs are different from those of long GRBs (for a recent review, see Lee \& Ramirez-Ruiz 2007).

The leading model for short GRBs involves the merging of a system composed of two collapsed objects, a double neutron star (DNS) or a black hole/neutron star binary. In those systems that evolve out of massive stars that were born in a binary system (we term these "primordial binaries"), the delay between formation and merging is dominated by the gravitational wave inspiral time, ranging from tens of Myr to a few Gyr (Perna \& Belczynski 2002), strongly dependent on the initial system separation. Short GRBs that result from them are expected to: (a) have a redshift 
distribution which broadly follows that of star formation and (b) drift away in some cases from the star-forming regions in which they were born, and merge outside, or in the outskirts, of galaxies (Belczynski et al. 2002).

A fraction of two collapsed object binaries may also form dynamically through binary exchange interactions in the core of globular clusters (Grindlay et al. 2006). For such a formation mechanism, the delay between star-formation and merging is driven by the cluster core collapse time, which is comparable to the Hubble time (Hopman et al. 2006). Therefore, short GRBs originating in dynamically formed double collapsed object binaries should go off at lower redshifts than short GRBs from primordial binaries (Guetta \& Piran 2005, 2006; Gal-Yam et al. 2006b; Hopman et al. 2006; Salvaterra et al. 2008). In another scenario, a fraction of the short GRBs is due to hyperflares from soft gamma-ray repeaters in the local universe (distances up to $\sim 100$ Mpc; Hurley et al. 2005; Tanvir et al. 2005; Frederiks et al. 2007; Mazets et al. 2008).

The above summary emphasizes that redshift determination, GRB position relative to the host galaxy and properties of the host galaxy are all crucial pieces of information for understanding short GRBs and discriminating among different models (Belczynski et al. 2006). One key issue in the study of short GRBs is the secure identification of the host galaxy. Indeed, several short GRBs afterglows have been detected only in X-rays and thus localized with a precision of a few arcseconds (whereas sub-arcesecond localizations are required to unambiguously reveal their host). The possibility that a sizeable fraction of short GRBs lie outside the light of their hosts, as predicted for both primordial and dynamically formed NS-NS/BH binaries, further complicates the identification process. Indeed some proposed short GRB associations with bright, nearby galaxies, based on some angular separation, might result from by-chance alignment.

So far, a dozen short GRBs were localized with subarcsecond precision and host galaxies were firmly detected with small offset. Only in a few cases (e.g. GRB 061201: Stratta et al. 2007; GRB 080503: Perley et al. 2008) no host galaxy was found down to $R \geq 26-28$ after the optical afterglow had faded. For other short GRBs at unknown redshift, a putative host galaxy was proposed with magnitude $R \sim 23-26$. Spectroscopy of the brightest four of these galaxies indicates that they lie at $0.4 \leq z \leq 1.1$. A comparison with field galaxy magnitudes suggests that the rest of the sample lies at $z \geq 1$ (Berger et al. 2007). The unambiguously localized hosts are both early- and late-type galaxies, with very different star formation rates and masses (Nakar 2007).

The association with early-type galaxies has provided clear evidence that at least a fraction of the short GRBs have progenitors related to an older stellar population than that of long GRBs, as expected from the compact binary system models. The nature of the progenitors of short GRBs that go off in star forming galaxies is still under debate. The possibility that short GRBs comprise different subclasses cannot be confirmed yet but neither excluded.

In this paper we present the results from an extensive campaign aimed at studying the optical afterglow of GRB 070707. This campaign monitored the decay of the optical afterglow evolution from about 0.45 days to more than one month after the burst. This is one of the best optical light curves for a short/hard GRB so far obtained. In Sect. 2 we report on previous results on GRB 070707. In Sect. 3 our observations and data analyses are discussed and in Sect. 4 our results are presented. In Sect. 5 we discuss our findings.

\section{GRB 070707}

GRB 070707 was detected in the 15-200 keV band with IBIS/ISGRI on board the INTEGRAL satellite (Mereghetti et al. 2003) on 2007 Jul. 7 at 16:08:21 UT, and initially classified as a long event (Beckmann et al. 2007). Subsequent analysis of the IBIS/ISGRI data determined a 2 '. 1 accurate position centered at $\mathrm{RA}(\mathrm{J} 2000)=17^{\mathrm{h}} 51^{\mathrm{m}} 00^{\mathrm{s}} .14, \operatorname{Dec}(2000)=-68^{\circ} 54^{\prime} 51^{\prime \prime} .8$, and revealed that the burst consisted of a single spike lasting about $1.1 \mathrm{~s}$ (Gotz et al. 2007). A refined, complete analysis of the INTEGRAL data of GRB 070707 has been presented by McGlynn et al. (2008), showing that its properties are all consistent with those of short GRBs: short duration $(0.8 \mathrm{~s})$, very small spectral lags $(20 \mathrm{~ms})$, and hard spectrum (photon index $\Gamma=1.2$ ). Konus-Wind also detected this burst (Golenetskii et al. 2007a), allowing the measurement of the broad-band fluence $\left(\sim 1.4 \times 10^{-6} \mathrm{erg} \mathrm{cm}^{-2}\right.$ in the $20 \mathrm{keV}-2 \mathrm{MeV}$ band $)$ and peak energy $(\sim 400 \mathrm{keV})$.

The Swift satellite began to observe GRB 070707 on 2007 Jul. 08 at 00:57:50 UT, i.e. about $9 \mathrm{~h}$ after the IBIS/ISGRI trigger. Swift/UVOT did not detect any optical afterglow down to a $3 \sigma$ limiting magnitude of $V=19.7$ (Schady et al. 2007). Swift/XRT found a faint, uncatalogued source inside the INTEGRAL error box, at RA(J2000) $=17^{\mathrm{h}} 50^{\mathrm{m}} 58^{\mathrm{s}} .49$, $\operatorname{Dec}(\mathrm{J} 2000)=-68^{\circ} 55^{\prime} 27^{\prime \prime} .1$ (positional uncertainty of 5'.4), with a $0.3-10 \mathrm{keV}$ flux of $2.4_{-1.4}^{+2.0} \times 10^{-13} \mathrm{erg} \mathrm{cm}^{-2} \mathrm{~s}^{-1}$ (Beardmore et al. 2007). Further Swift/XRT observations, carried out about 4.9 days after the burst, could not detect the source down to a ten times lower flux level. This confirmed that the Swift/XRT source was indeed the afterglow of GRB 070707 (Beardmore \& Parsons 2007).

ESO-VLT observations, carried out starting about half a day after the burst, revealed the presence of a variable $R$-band source inside the Swift/XRT error circle, at RA $(J 2000)=17^{\mathrm{h}} 50^{\mathrm{m}} 58.55$, $\operatorname{Dec}(\mathrm{J} 2000)=-68^{\circ} 55^{\prime} 27^{\prime \prime} .2$ (0.'3 error; Piranomonte et al. 2007; D'Avanzo et al. 2007b). No further observations of the afterglow of GRB 070707 have been reported so far.

\section{Observations and data analysis}

We observed GRB 070707 with the ESO-VLT at eight different epochs starting about $11 \mathrm{~h}$ after the burst. Observations were carried out using the FORS1, ISAAC and NACO cameras. All nights were clear, with seeing in the $0.5^{\prime \prime}-1.0^{\prime \prime}$ range. Image reduction was carried out following standard procedures: subtraction of the bias frame and division by the flat frame. Point spread function (PSF) and aperture photometry were obtained by using the Daophot II (Stetson 1987) in the ESO-MIDAS ${ }^{1}$ package for all objects in the field. Photometric calibration was based on Landolt standard stars, observed in different nights. In order to minimize systematic effects, we performed differential photometry with respect to a selection of local isolated and non-saturated standard stars. Astrometric solutions were computed by using the USNO-B1.0 catalogue ${ }^{2}$.

All our VLT/FORS spectra were acquired with the $300 \mathrm{~V}$ grism, covering the 4000-9000 ̊ wavelength range ( $7 \AA$ FWHM resolution). We always used a $1^{\prime \prime}$ slit. The extraction of the spectra was performed with the $\mathrm{IRAF}^{3}$ software package.

\footnotetext{
1 http://www . eso.org/projects/esomidas/

2 http://www .nofs . navy .mil/data/fchpix/

3 IRAF is distributed by the National Optical Astronomy Observatories, which are operated by the Association of the Universities for Research in Astronomy, Inc., under cooperative agreement with the National Science Foundation.
} 
Table 1. VLT observation log for GRB 070707. Magnitudes are not corrected for Galactic absorption. Upper limits are given at $3 \sigma$ confidence level.

\begin{tabular}{llrrrrr}
\hline \hline $\begin{array}{l}\text { Mean time } \\
(\mathrm{UT})\end{array}$ & $\begin{array}{l}\text { Exposure time } \\
(\mathrm{s})\end{array}$ & $\begin{array}{r}t-t_{0} \\
(\text { days })\end{array}$ & $\begin{array}{c}\text { Seeing } \\
\left({ }^{\prime \prime}\right)\end{array}$ & Instrument & Magnitude & Filter / Grism \\
\hline 2007 Jul. 08.12988 & $10 \times 120$ & 0.45742 & 0.9 & VLT/FORS1 & $23.05 \pm 0.02$ & $R$ \\
2007 Jul. 09.07984 & $10 \times 120$ & 1.40738 & 1.0 & VLT/FORS1 & $23.86 \pm 0.05$ & $R$ \\
2007 Jul. 09.23005 & $1 \times 180$ & 1.55759 & 1.0 & VLT/FORS1 & $24.07 \pm 0.12$ & $R$ \\
2007 Jul. 10.14491 & $10 \times 120$ & 2.47245 & 0.7 & VLT/FORS1 & $25.33 \pm 0.08$ & $R$ \\
2007 Jul. 10.21523 & $20 \times 3 \times 30$ & 2.54277 & 0.7 & VLT/ISAAC & $>23.6$ & $J$ \\
2007 Jul. 11.13697 & $20 \times 180$ & 3.46721 & 0.5 & VLT/FORS1 & $26.62 \pm 0.18$ & $R$ \\
2007 Jul. 12.17370 & $17 \times 300$ & 4.50124 & 0.8 & VLT/FORS1 & $26.81 \pm 0.22$ & $R$ \\
2007 Jul. 15.21785 & $18 \times 300$ & 7.54539 & 0.6 & VLT/FORS1 & $27.39 \pm 0.22$ & $R$ \\
2007 Jul. 19.12752 & $20 \times 300$ & 11.45506 & 0.6 & VLT/FORS1 & $27.21 \pm 0.20$ & $R$ \\
2007 Aug. 15.07151 & $20 \times 300$ & 38.38355 & 0.6 & VLT/FORS1 & $27.15 \pm 0.29$ & $R$ \\
2007 Sep. 28.03125 & $20 \times 3 \times 60$ & 81.95974 & 0.5 & VLT/NACO & $>22.7$ & $K$ \\
\hline 2007 Jul. 09.32508 & $5 \times 2400$ & 1.65262 & 1.0 & VLT/FORS1 & - & $300 V+G G 375$ \\
2007 Jul. 13.15960 & $2 \times 900$ & 5.48714 & 0.8 & VLT/FORS1 & - & $300 V+G G 375$ \\
\hline
\end{tabular}

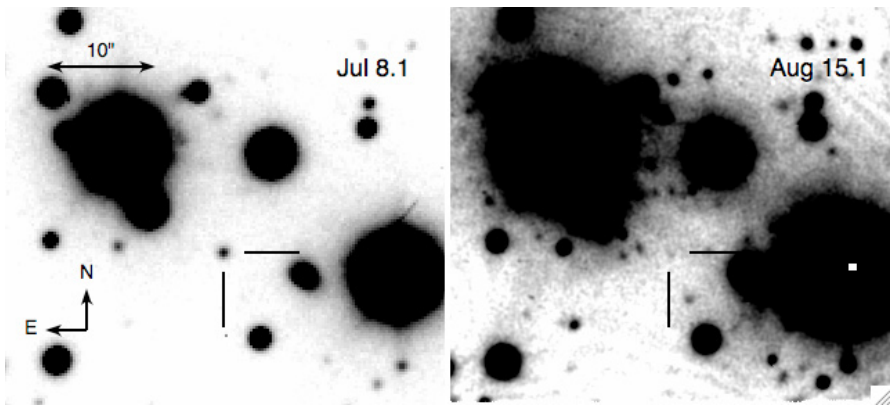

Fig. 1. $R$-band image of the optical afterglow (left panel) and of the host galaxy (right panel) of GRB 070707.

Table 2. Results of the optical-light curve fitting with a Beuermann function (Beuermann et al. 1999) freezing the smoothness parameter $\kappa=1$. Errors are at $1 \sigma$ with all parameters free to vary.

\begin{tabular}{ccccc}
\hline \hline$\alpha_{1}$ & $\alpha_{2}$ & $t_{\mathrm{b}}$ (days) & Host $R$ magnitude & $\chi^{2} /$ d.o.f. \\
\hline $0.44_{-0.21}^{+0.08}$ & $5.3_{-0.8}^{+0.9}$ & $1.82_{-0.25}^{+0.13}$ & $27.3 \pm 0.13$ & $1.96 / 4$ \\
\hline
\end{tabular}

Wavelength and flux calibration of the spectra were obtained by using helium-argon lamp and observing spectrophotometric stars. A complete log of our observations, together with the results of our analysis, is reported in Table 1.

\section{Results}

\subsection{VLT photometry}

The optical light curve appears to have had an initial slow decay, which got significantly steeper beginning 1-2 days after the GRB. At late times, a constant flux was observed, indicating a dominant contribution from the host galaxy (Fig. 1). We fitted the light curve with a model comprising a broken power law behaviour, representing the afterglow, plus a constant, representing the host. We adopted the usual Beuermann function (Beuermann et al. 1999) to model a transition from a shallow to a steeper decay: $F(t)=F_{0} /\left[\left(t / t_{\mathrm{b}}\right)^{\kappa \alpha_{1}}+\left(t / t_{\mathrm{b}}\right)^{\kappa \alpha_{2}}\right]^{1 / \kappa}$, where $\alpha_{1}$ and $\alpha_{2}$ are the early- and late-time decay slopes, $t_{\mathrm{b}}$ is the break time, and $\kappa$ is the smoothness parameter. In the case of GRB 070707, given the relatively low number of data points, we first froze $\kappa=1$ in our fit. In Table 2 we report the best-fit parameters.
Table 3. Results of the optical-light curve fitting with the "pulse function". Errors are at $1 \sigma$ with all parameters free to vary.

\begin{tabular}{cccc}
\hline \hline$\tau$ (days) & $t_{0}$ (days) & Host $R$ magnitude & $\chi^{2} /$ d.o.f. \\
\hline $0.52_{-0.07}^{+0.07}$ & $-0.06_{-0.29}^{+0.23}$ & $27.3 \pm 0.24$ & $2.28 / 5$ \\
\hline
\end{tabular}

When $\kappa$ was allowed to vary, the break time was only weakly constrained and the slope $\alpha_{1}$ could also take negative values (i.e. the light curve could have also be initially rising and peak around 0.5 days). In any case the late time decay remained steep, with a lower limit $\alpha_{2} \geq 3$ valid for any $\kappa$.

We note that different functional forms cannot be excluded for the light curve, given the sparse coverage at early times. For example a model consisting of a linear rise followed by an exponential decay $F(t)=F_{1}\left(t-t_{0}\right) \mathrm{e}^{-\left(t-t_{0}\right) / \tau}$ (we refer to this as the "pulse function") provided a satisfactory fit to the data (even for $t_{0}=0$, i.e. the origin of time coincident with the high-energy event; Table 3). The best fit models with the Beuermann function (with $\kappa=1$ ) and the "pulse function" are shown in Fig. 2.

\subsubsection{VLT spectroscopy}

After the identification of the optical counterpart (Piranomonte et al. 2007) we took an optical spectrum of the afterglow (about 1.7 days after the burst; see Table 1), consisting of five frames with a total exposure time of $200 \mathrm{~min}$. Due to the rapid decay of the afterglow, the object was clearly visible only in the first two frames. As a consequence the total spectrum has a mediocre signal-to-noise ratio. Neither emission nor absorption lines are visible over a weak continuum in the 4200-9000 $\AA$ range. The detection of the continuum, on the other hand, allows us to put some constraints on the redshift of this event. The most conservative limit on the redshift is given by the lack of Lyman limit suppression down to $4200 \AA$, which implies $z<3$.6. The lack of observed Lyman- $\alpha$ forest provides a tighter constraint $z \lesssim 2.5$, although at such redshift the density of the IGM is relatively low, making this limit less robust.

Many galaxies (a few tens) are clearly visible in our VLT images of GRB 070707. In order to check for the possible presence of a cluster we obtained optical spectra of five galaxies in the field and determined their redshifts (Table 4 and Fig. 3). These spectra were taken on 2007 Jul. 9th and 13th (Table 1). The redshift gap between G1, G2 and G3 (0.3547-0.3780) is very large and corresponds to a velocity difference of $7000 \mathrm{~km} \mathrm{~s}^{-1}$ with a 


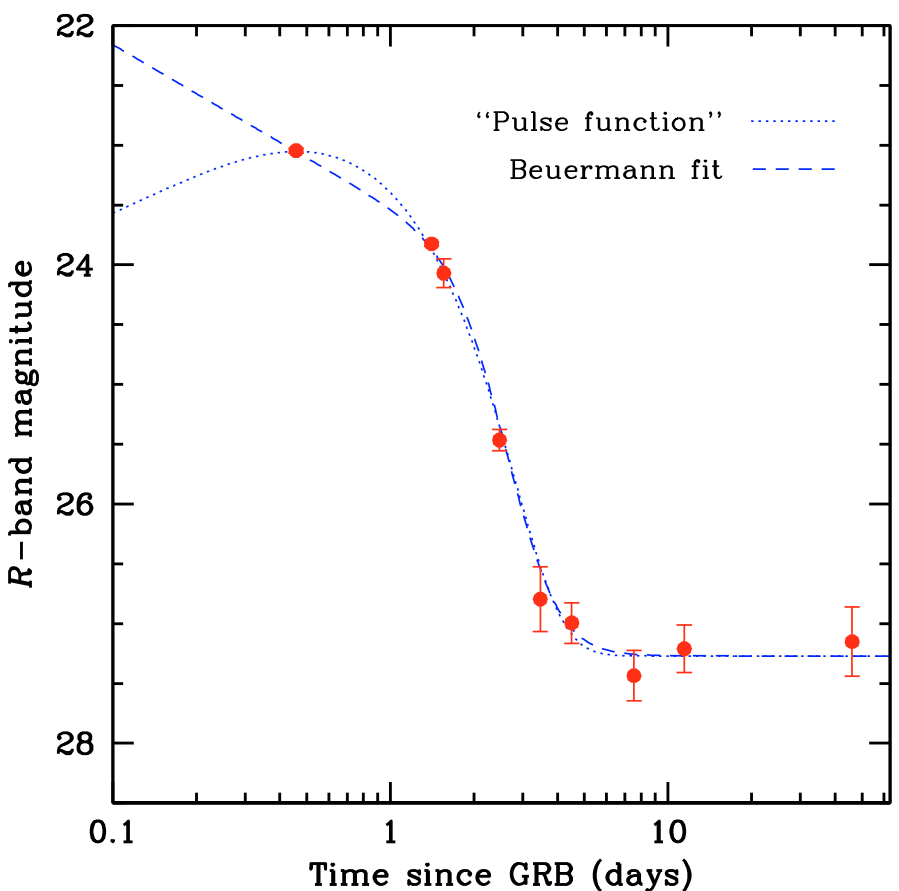

Fig. 2. $R$-band light curve of the GRB 070707 afterglow. The dashed line represents the fit obtained using a Beuermann function (with $\kappa=1$ ), while the dotted line shows the fit with the "pulse function" (see text).

Table 4. Properties of five galaxies in the field of GRB 070707 (see Fig. 3 and Sect. 4 for details).

\begin{tabular}{ccccc}
\hline \hline ID & RA (J2000) & Dec (J2000) & $R$ magnitude & Redshift \\
\hline G1 & $17: 50: 58.55$ & $-68: 53: 35.0$ & 21.1 & 0.3547 \\
G2 & $17: 50: 58.40$ & $-68: 53: 01.5$ & 20.5 & 0.3780 \\
G3 & $17: 51: 04.73$ & $-68: 54: 27.0$ & 20.2 & 0.3627 \\
G4 & $17: 51: 00.67$ & $-68: 55: 01.8$ & 17.6 & 0.213 \\
G5 & $17: 50: 58.97$ & $-68: 55: 12.8$ & 20.5 & 0.667 \\
G6 & $17: 50: 57.18$ & $-68: 55: 29.4$ & 20.4 & 0.2394 \\
\hline
\end{tabular}

dispersion of $3500 \mathrm{~km} \mathrm{~s}^{-1}$. This value is considerably higher than those measured in clusters of galaxies; therefore we consider very unlikely that these galaxies belong to a cluster. The object labelled as G6 is the closest to the position of the GRB, with a separation of 7!'8. A qualitative analysis of the optical spectrum of galaxy G6 (Fig. 4) reveals that it is a starburst galaxy at $z=0.2394$, as indicated by the presence of prominent nebular lines. If the GRB were also occurring at this redshift, the projected offset would be about $29.3 \mathrm{kpc}$, and its host galaxy would have an absolute visual magnitude of $M_{\mathrm{V}} \sim-13.2$, a value similar to dwarf galaxies of the Local Group, such as Fornax, but still much brighter than a globular cluster.

\section{Discussion}

As with a number of other short GRBs, the nearly unconstrained redshift of GRB 070707 remains an important limiting factor. In the peak energy vs. isotropic-equivalent energy diagram (Fig. 5), GRB 070707 should lie on the thick solid curve marked with selected values of the redshift. For the range of possible redshifts, the position of GRB 070707 would be in broad agreement with that of other short GRBs with known redshift and peak energy, which notoriously do not follow the so-called "Amati-relation" of long GRBs (Amati 2006).

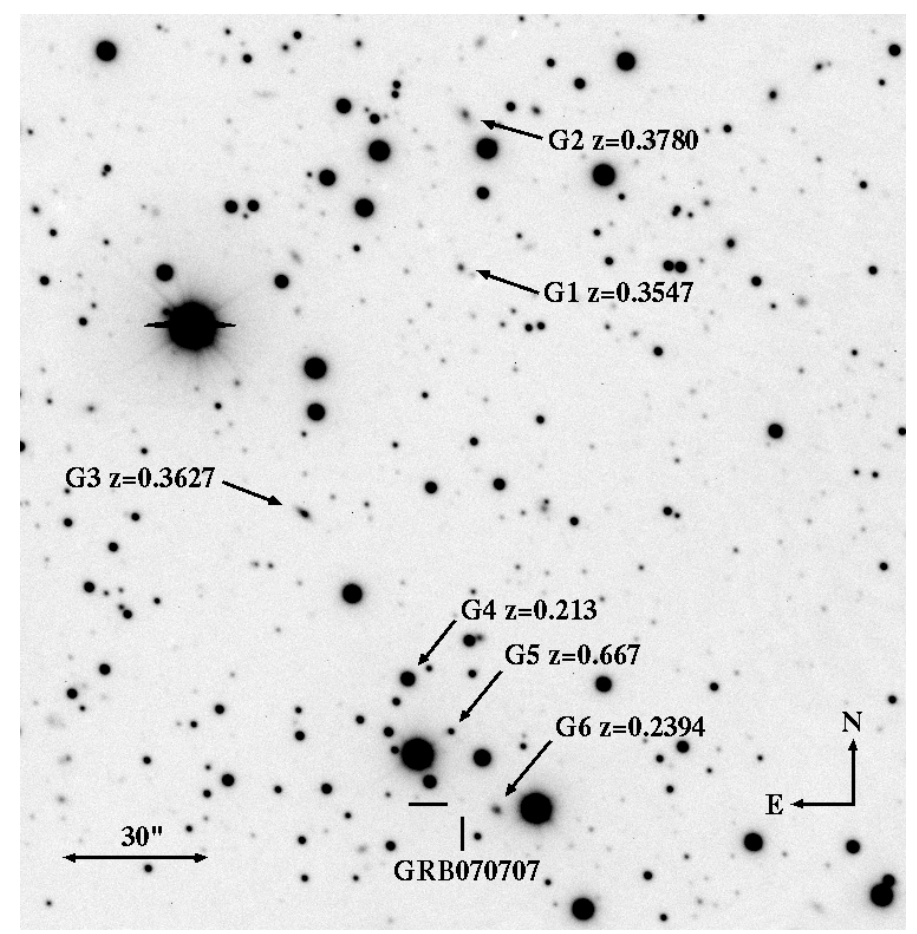

Fig. 3. $R$-band image of the field of GRB 070707. The galaxies whose redshifts have been reported in Table 4 are marked, together to the position of GRB 070707. The field is about $3^{\prime} \times 3^{\prime}$ wide.

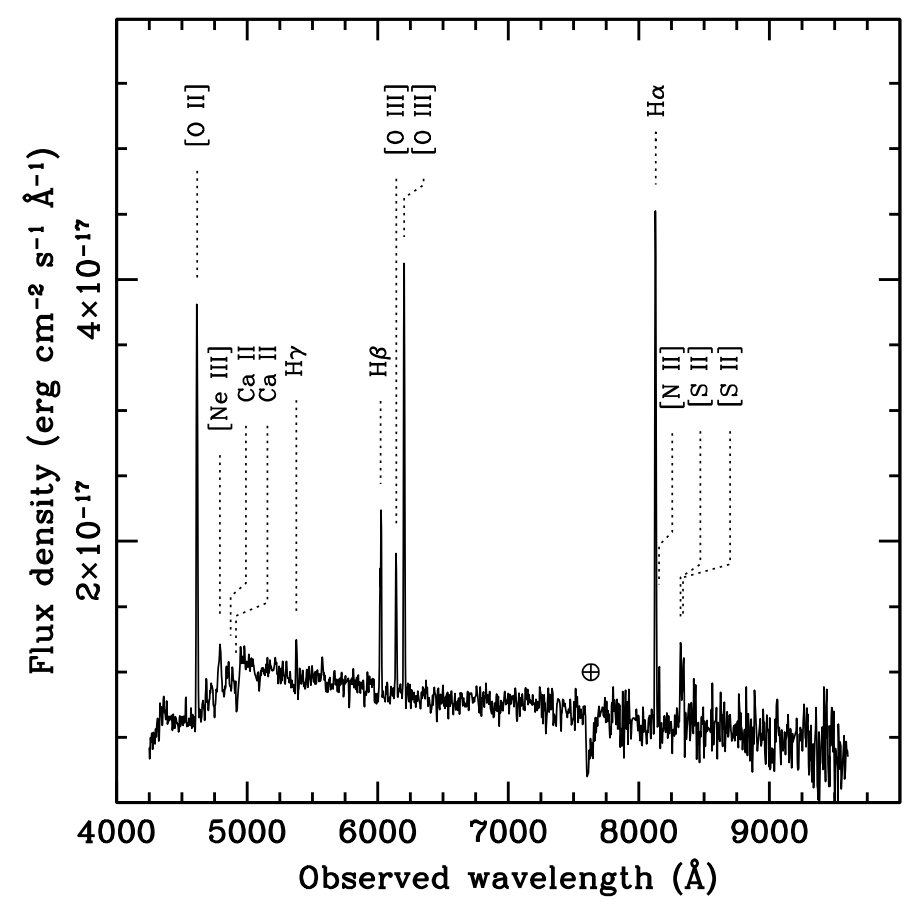

Fig. 4. VLT-FORS2 spectrum of galaxy G6 (see Fig. 3) at $z=0.2394$.

Whichever its redshift, the host of GRB $070707(R \sim 27.3)$ is the faintest ever detected for a short GRB, with a magnitude comparable to that of long GRB hosts at high redshift (e.g. Wainwright et al. 2007; Fruchter et al. 2006). In past works (e.g. Bloom et al. 2007; Stratta et al. 2007; Levan et al. 2007), there has been discussion about the possibility to find short GRBs not spatially coincident with a host galaxy. In some cases, nearby, bright objects were proposed to be the GRB host based just on angular proximity. The case of GRB 070707 shows that caution 


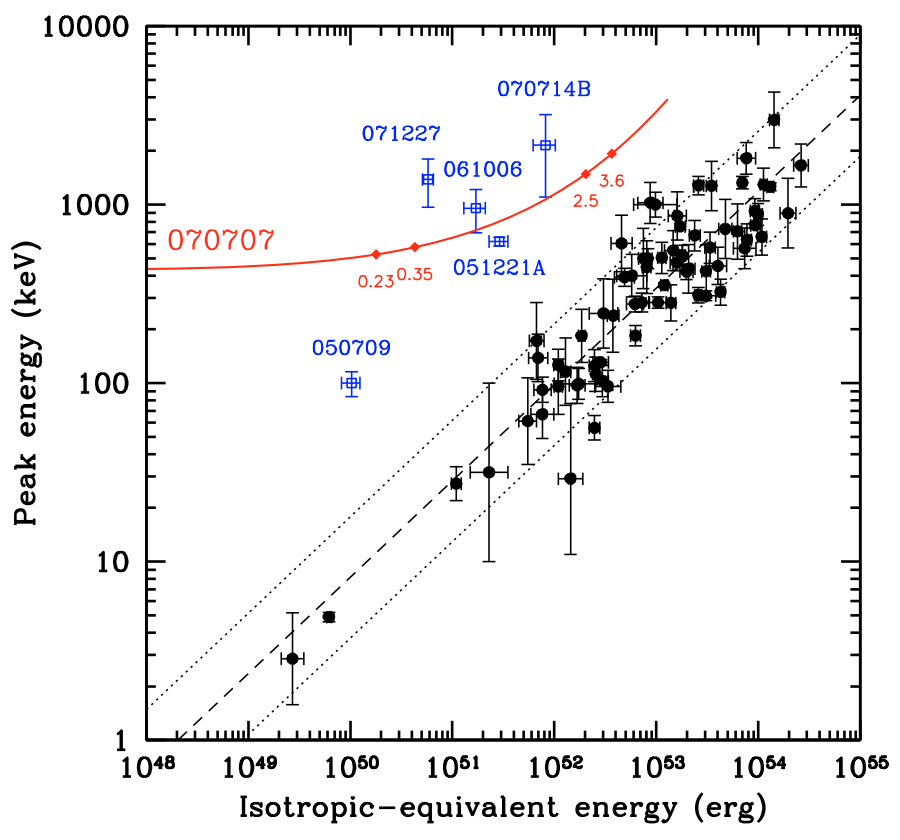

Fig. 5. Location of GRB 070707 in the plane peak energy vs. isotropic-equivalent energy. The thick solid line shows the position of GRB 070707 as a function of redshift, with the diamonds indicating specific values discussed in this paper. Filled circles represent longduration GRBs (from Amati et al. 2008), and the diagonal lines indicate the best-fit Amati relation (dashed) and the $2 \sigma$ contours (dotted). Empty squares indicate other short-duration events with known redshift and peak energy (Amati 2006; Golenetskii et al. 2006; Ohno et al. 2007; Golenetskii et al. 2007b).

is needed. Without deep VLT images, it might have been tempting to associate GRB 070707 with the closeby galaxy G6, which is certainly not the GRB host. With such a large magnitude contrast between the afterglow and the host, many more galaxies of short GRBs may be just fainter than the fluxes probed by shallower exposures. While this is consistent with the suggestion that a sizeable fraction of short GRBs reside at redshift larger than $z \sim 1$ (Berger et al. 2007), this may also indicate that some short GRBs go off inside low-luminosity galaxies at low redshift.

The sparse data on the host galaxy of GRB 070707 do not allow for a detailed analysis. At the maximum allowed redshift $z=3.6$, the host would have an absolute magnitude $M_{\mathrm{AB}}=$ -18.6 (at rest-frame wavelength $\lambda \approx 1500 \AA$ ). This is more that 2 mag fainter than the Schechter luminosity at this redshift $\left(M_{\mathrm{AB}}^{*}=-20.7\right.$; Gabasch et al. 2004). We can thus firmly set $L<(1 / 6) L_{*}$ at any redshift, implying that the host was intrinsically faint. Our late-time NIR upper limit $(K>22.7)$ implies $R-K<4.3$ and hence can rule out a bright, red host, such as an extremely red object (ERO) or a moderate-redshift elliptical. GRB 070707 hence confirms that short GRBs can explode inside faint and possibly extremely faint systems. Short GRB hosts indeed exhibit a wide range of luminosities.

Our spectrum did not show any clear absorption feature, which is quite possibly due to the low signal-to-noise and/or limited covered wavelength range. It is however interesting to note that a featureless spectrum has been reported also for the afterglow of the short GRB 061201 (Stratta et al. 2007). If confirmed by further, better-quality observations, this fact may provide hints for a lower density of short GRB environments compared to those of long-duration events.

Further information on the GRB progenitor comes from the optical light curve of the GRB 070707 afterglow, which decayed very steeply starting $\sim 1.5$ days from the burst. The power law decay index $\alpha$ is constrained by our fits to be steeper than 3, or may even be exponential. Assuming that the optical afterglow emission came from the forward shock, as commonly supposed in the fireball model for GRB afterglows, the steepening of the optical light curve could be interpreted as a jet break. However the index measured for GRB 070707 is too steep to be explained in terms of jetted emission. In fact this would require $\alpha_{2}=p$ (Rhoads 1999), where $p$ is the electron energy distribution index. Such a steep decay could be marginally consistent with the post-break phase only by adopting a very soft electron energy distribution $(p>3)$. Such large $p$ values have never been found in GRB afterglows, both from theoretical and empirical investigations (e.g. Panaitescu 2005; Shen et al. 2006; Tagliaferri et al. 2006; Kann et al. 2006). In particular, values of $p<3$ are inferred based on afterglow spectra, in which case the analysis is more robust than when relying on the temporal behaviour. Moreover, the magnitude of the steepening from the pre- to the post-break decay would be too pronounced for a jet-break interpretation in GRB 070707.

The steepness of the observed decay might be difficult to reconcile with forward shock emission even just for causality reasons. The fastest possible decay is the so-called high-latitude emission, which occurs when the fireball emission stops abruptly and the observers see photons coming from the wings of the emitting surface. In this case, $\alpha=2+\beta$, where $\beta$ is the afterglow spectral index (Kumar \& Panaitescu 2000). The observed upper limit in the $J$ band (Table 1) allows us to derive $R-J<2.1$ on Jul. 10.2 UT, which corresponds to $\beta<1.95$. This would still be consistent with the high-latitude interpration. A stronger limit on $\beta$ can however be inferred by using the X-ray data. Assuming a synchrotron spectrum, the optical spectral index can never be softer than the optical-to-X-ray spectral index $\beta_{\mathrm{OX}}$. For GRB 070707, the observed X-ray flux (Beardmore \& Parsons 2007) implies $\beta_{\mathrm{OX}}=0.75_{-0.09}^{+0.13}$. High-latitude emission from a source with such a spectrum cannot decay faster than $t^{-2.75}$, hence effectively ruling out such possibility for GRB 070707.

A viable alternative to explain the steep decay of GRB 070707 is that of a long-lived central engine. In this case, as discussed by several authors (e.g. Zhang et al. 2006), the decay index should be computed after setting the zero time $t_{0}$ to the end of the extended activity phase. The intrisic decay slope would therefore be shallower than the observed value, eliminating the causality problem. The very steep decay of GRB 070707 thus would provide further evidence that the inner engine powering short GRBs is working for a much longer time than the observed gamma-ray emission. The optical light curve of GRB 070707 might be produced, for example, by a large flare, as also argued for GRB 050724 (Barthelmy et al. 2005; Malesani et al. 2007).

Finally, we mention that the optical light curve of GRB 070707 could also be explained within the context of cannonball model (Dado \& Dar 2008; Dado et al. 2008, and references therein). The emitting region in this case has angular dimension well below $1 / \Gamma$, and therefore the causality constraints are much more relaxed.

Acknowledgements. We thank an anonymous referee for comments. We thank D. A. Kann and A. Dar for useful discussion. Part of this work was supported by MIUR COFIN-03-02-23 and INAF/PRIN 270/2003 and ASI contracts ASI/I/R/039/04 and ASI/I/R/023/05/0. S.D.V. is supported by SFI. The Dark Cosmology Centre is funded by the Danish National Research Foundation. A.P.B. is supported by STFC. 


\section{References}

Amati, L. 2006, MNRAS, 372, 233

Amati, L., Guidorzi, C., Frontera, F., et al. 2008, MNRAS, submitted [arXiv:0805.0377]

Barthelmy, S. D., Chincarini, G., Burrows, D. N., et al. 2005, Nature, 438, 994 Beardmore, A. P., \& Parsons, A. 2007, GCN, 6626

Beardmore, A. P., Page, K. L., \& Parsons, A. 2007, GCN, 6610

Beckmann, V., Ricci, C., Beck, M., et al. 2007, GCN, 6605

Belczynski, K., Bulik, T., \& Kalogera, V. 2002, ApJ, 571, 147

Belczynski, K., Perna, R., Bulik, T., et al. 2006, 648, 1110

Berger, E. 2007, ApJ, 670, 1254

Berger, E. 2008, [arXiv:0805.0306]

Berger, E., Price, P. A., Cenko, S. B., et al. 2005, Nature, 438, 988

Berger, E., Fox, D. B., Price, P. A., et al. 2007, ApJ, 664, 1000

Beuermann, K., Hessman, F. V., Reinsch K., et al. 1999, A\&A, 352, L26

Bloom, J. S., Perley, D. A., Chen, H.-W., et al. 2007, ApJ, 654, 878

Covino, S., Malesani, D., Israel, G. L., et al. 2006, A\&A, 447, 5

D’Avanzo, P., Piranomonte, S., Stella, L., \& Chincarini, G. 2007a, GCN, 6609

D’Avanzo, P., Piranomonte, S., Vergani, S. D., Christensen, L., \& Amico, P. 2007b, GCN, 6613

Dado, S., \& Dar, A. 2008, [arXiv:0807.1962]

Dado, S., Dar, A., De Rújula, A., \& Plaga, R. 2008, ApJ, 678, 353

Della Valle M., Chincarini, G., Panagia, N., et al. 2006, Nature, 444, 1050

Frederiks, D. D., Palshin, V. D., Aptekar, R. L., et al. 2007, Ast. Lett., 33, 19

Fox, D. B., \& Mészáros, P. 2006, New J. Phys., 8, 199

Fruchter, A. S., Levan, A. J., Strolger, L., et al. 2006, Nature, 441, 463

Fynbo, J. P. U., Watson, D., Thöne, C. C., et al. 2006, Nature, 444, 1047

Gabasch, A., Bender, R., Seitz, S., et al. 2004, A\&A, 421, 41

Gal-Yam, A., Fox, D. B., Price, P. A., et al. 2006, Nature, 444, 1053

Gal-Yam, A., Ofek, E. O., Poznanski, D., et al. 2006, ApJ, 639, 331

Gehrels, N., Sarazin, C., O’Brien, P. T., et al. 2005, Nature, 437, 851

Golenetskii, S., Aptekar, R., Mazets, E., et al. 2006, GCN, 5710

Golenetskii, S., Aptekar, R., Mazets, E., et al. 2007a, GCN, 6615

Golenetskii, S., Aptekar, R., Mazets, E., et al. 2007b, GCN, 7155

Gotz, D., Beckmann, V., Mereghetti, S., \& Paizis, A. 2007, GCN, 6607

Grindlay, J., Portegies Zwart, S., \& McMillan, S. 2006, Nat. Phys., 2, 116

Guetta, D., \& Piran, T. 2005, A\&A, 435, 421

Guetta, D., \& Piran, T. 2006, A\&A, 453, 823
Hjorth, J., Watson, D., Fynbo, J. P. U., et al. 2005, Nature, 437, 859

Hopman, C., Guetta, D., Waxman, E., \& Portegies, Z. S. 2006, ApJ, 643, 91

Hurley, K., Boggs, S. E., Smith, D. M., et al. 2005, Nature, 434, 1098

Kann, D. A., Klose, S., \& Zeh, A. 2006, ApJ, 641, 993

Kann, D. A., Klose, S., Zhang, B., et al. 2008, ApJ, submitted [arXiv: 0804.1959]

Kouveliotou, C., Meegan, C. A., Fishman, G. J., et al. 1993, ApJ, 413, 101

Kumar, P., \& Panaitescu, A. 2000, ApJ, 541, L41

Lee, W. H., \& Ramirez-Ruiz, E. 2007, New. J. Phys., 9, 17

Levan, A. J., Jakobsson, P., Hurkett, C., et al. 2007, MNRAS, 378, 1439

Malesani, D., Covino, S., D'Avanzo, P., et al. 2007, A\&A, 473, 77

Mazets, E. P., Golenetskii, S. V., Ilinskii, V. N., et al. 1981, Ap\&SS, 80, 3

Mazets E. P., Aptekar R. L., Cline T. L., et al. 2008, ApJ, 680, 545

McGlynn, S., Foley, S., McBreen, S., et al. 2008, A\&A, 486, 405

Mei, S., Holden, B. P., Blakeslee, J., P., et al. 2006, ApJ, 644, 759

Mereghetti, S., Gotz, D., Borkowski, J., et al. 2003, A\&A, 411, L291

Muriel H., Quintana H., Infante L., et al. 2002, AJ, 124, 1934

Nakar, N. 2007, Phys. Rep., 442, 166

Norris J. P, Cline T. L., Desai U. D., \& Teegarden B. J. 1984, Nature, 308, 434

Ohno, M., Uehara, R., Takahashi, T., et al. 2007, GCN, 6638

Panaitescu, A. 2005, MNRAS, 363, 1409

Perley, D. A., Bloom, J. S., Levan, A. J., et al. 2008, GCN, 7749

Perna, R., \& Belczynski, K. 2002, ApJ, 570, 252

Piranomonte, S., Vergani, S. D., D’Avanzo, P., \& Tagliaferri, G. 2007a, GCN, 6612

Rhoads, J. E. 1999, ApJ, 525, 737

Salvaterra, R., Cerutti, A., Chincarini, G., et al. 2008, MNRAS, 388, L6

Schady, P., \& Parsons, P. 2007, GCN, 6611

Shen R., Kumar P., \& Robinson E. L. 2006, MNRAS, 371, 1441

Stetson, P. B. 1987, PASP, 99, 191

Stratta, G., D'Avanzo, P., Piranomonte, S., et al. 2007, A\&A, 474, 827

Struble, M. F., \& Rood, H. J. 1999, AJSS, 125, 35

Tagliaferri G., Malesani D., Vergani S. D., et al. 2006, NCim B, 121, 1163

Tanvir, N. R., Chapman, R., Levan, A. J., \& Priddey, R. S. 2005, Nature, 438, 991

Tavani, M. 1996, Phys. Rev. Lett., 76, 3478

Wainwright, C., Berger, E., \& Penprase, B. E. 2007, ApJ, 657, 367

Zhang, B., \& Mészáros P. 2004, IJMPA, A19, 2385

Zhang, B., Fan, Y., Dyks, J., et al. 2006, ApJ, 642, 354 